\title{
Association of GBA Genotype With Motor and Functional Decline in Patients With Newly Diagnosed Parkinson Disease
}

Jodi Maple-Grødem, PhD, Ingvild Dalen, PhD, Ole-Bjørn Tysnes, MD, PhD, Angus D. Macleod, MRCP, PhD, Lars Forsgren, MD, PhD, Carl E. Counsell, MRCP, MD, and Guido Alves, MD, PhD

Neurology ${ }^{\circledR}$ 2021;96:e1036-e1044. doi:10.1212/WNL.0000000000011411
Correspondence Dr. Maple-Grødem jodi.maple@uis.no

\section{Abstract \\ Objective}

To establish the significance of glucocerebrosidase gene (GBA) carrier status on motor impairment in a large cohort of patients with incident Parkinson disease (PD).

\section{Methods}

Three European population-based studies followed 528 patients with PD from diagnosis. A total of 440 with genomic DNA from baseline were assessed for GBA variants. We evaluated motor and functional impairment annually using the Unified Parkinson's Disease Rating Scale (UPDRS) motor and activities of daily living (ADL) sections. Differential effects of classes of GBA variants on disease progression were evaluated using mixed random and fixed effects models.

\section{Results}

A total of 387 patients with idiopathic disease (age at baseline $70.3 \pm 9.5$ years; $60.2 \%$ male) and 53 GBA carriers (age at baseline $66.8 \pm 10.1$ years; $64.2 \%$ male) were included. The motor profile of the groups was clinically indistinguishable at diagnosis. GBA carriers showed faster annual increase in UPDRS scores measuring ADL (1.5 point per year, 95\% confidence interval [CI] 1.1-2.0) and motor symptoms (2.2 points per year, 95\% CI 1.3-3.1) compared to noncarriers (ADL, 1.0 point per year, $95 \%$ CI $0.9-1.1, p=0.003$; motor, 1.3 point per year, $95 \%$ CI $1.1-1.6, p=0.007)$. Simulations of clinical trial designs showed that recruiting only GBA carriers can reduce trial size by up to $65 \%$ compared to a trial recruiting all patients with PD.

\section{Conclusion}

GBA variants are linked to a more aggressive motor disease course over 7 years from diagnosis in patients with PD. A better understanding of PD progression in genetic subpopulations may improve disease management and has direct implications for improving the design of clinical trials. 


\section{Glossary}

$\mathrm{ADL}=$ activities of daily living; $\mathrm{CI}=$ confidence interval; $\mathbf{G B A}=$ glucocerebrosidase gene; $\mathrm{H} \& \mathrm{Y}=$ Hoehn \& Yahr; LED = levodopa-equivalent dose; MMSE = Mini-Mental State Examination; PD = Parkinson disease; PPMI = Parkinson's Progression Markers Initiative; UPDRS = Unified Parkinson's Disease Rating Scale.

Parkinson disease $(\mathrm{PD})$ is a neurodegenerative disorder defined by the presence of motor symptoms and signs, though nonmotor symptoms are common in all stages of the disease. The disease course is typically progressive and in the advanced stages, motor impairment results in significant disability.

The glucocerebrosidase gene (GBA) encodes the lysosomal enzyme $\beta$-glucocerebrosidase. Mutations and polymorphisms in GBA are estimated to be found in up to $12 \%$ of patients with PD of European descent, and mutations in $15 \%-20 \%$ of Ashkenazi Jewish cases, making GBA the most significant genetic risk factor for PD. ${ }^{1-5}$ GBA-associated PD has been shown to manifest at a younger age and with a lower median survival time from diagnosis compared to idiopathic PD. ${ }^{6-8}$ Further, GBA mutation status is an independent risk factor for cognitive impairment. ${ }^{3,5,9-11}$

PD is primarily a movement disorder yet few studies have analyzed the effect of GBA carrier status on motor decline in early PD. In this study, we address this using longitudinal data from 3 deeply phenotyped population-based studies of incident PD in Northern Europe to comprehensively survey the progression of motor impairment in early PD. By determining the role of GBA variants in the evolution of motor and functional decline, we provide important new insights into the distinct clinical profile of GBA-associated PD, with importance for both more individualized patient care and more efficient clinical trial design.

\section{Methods}

\section{Cohorts, Approvals, and Patient Consents}

The participants in this study take part in the Norwegian ParkWest study, the Swedish NYPUM study, and the Scottish PINE study, 3 prospective population-based longitudinal incidence studies of PD (recruitment running between 2002 and 2009) with similar study design. ${ }^{12-15}$ A total of 212 patients were enrolled in the ParkWest study, 211 in the PINE study, and 182 in the NYPUM study. The consent rate to participation among incident patents was $80 \%$ in the ParkWest study, 94\% in the PINE study, and $94 \%$ in the NYPUM study. The patients are under continued follow-up, and only those with a confirmed clinical or pathologic (if performed postmortem) diagnosis of PD according to the UK Brain Bank criteria at their latest or final clinical visit were included. Since enrollment, 70 had a diagnosis other than PD during follow-up. Furthermore, 57 declined genotyping, 31 have no available DNA sample or DNA was not extractable, and 7 did not consent to follow-up. The remaining 440 patients were eligible for this study and the 7-year clinical visits were complete.

\section{Standard Protocol Approvals, Registrations, and Patient Consents}

The Western Norway Regional Committee for Medical and Health Research Ethics, the Regional Ethics Review Board in Umeå, and the Multi-Centre Research Ethics Committee for Scotland approved the respective studies. Written informed consent was obtained from all patients participating in the study (consent for research).

\section{Clinical Assessment}

The clinical assessments have been described in detail and the same procedures were followed in each cohort. ${ }^{12-15}$ Briefly, a study neurologist performed general medical and neurologic examinations and semi-structured interviews at baseline to obtain medical, drug, and family history (defined as the report of a first- or second-degree relative with PD). We reassessed the patients at regular follow-up visits and offered home visits to those unable or not willing to meet at the clinic to minimize attrition bias. We used the Hoehn \& Yahr (H\&Y) scale to rate disease stage ${ }^{16}$ and the Unified Parkinson's Disease Rating Scale (UPDRS) ${ }^{17}$ sections II (activities of daily living [ADL]) and III (motor examination). In addition to overall motor impairment, we assessed the severity of specific motor features of PD by the summation of the relevant UPDRS III items as follows: tremor (items 20-21), rigidity (items 22), bradykinesia (items 23-26, 31), and axial impairment (items 27-30). Assessment of nonmotor features included the MiniMental State Examination (MMSE). Antiparkinsonian treatment was prescribed and adjusted throughout the study by a study neurologist according to best clinical judgment. We calculated levodopa-equivalent doses (LED) in accordance with published recommendations. ${ }^{18}$

\section{Genetic Analysis}

The presence of GBA mutations (rs76763715/N370S, rs421016/L444P, and rs781152868/Y135C) or GBA polymorphisms (rs2230288/E326K, rs75548401/T369M, and rs369068553/V460L) in the study population has been described in detail. ${ }^{5}$ Briefly, genomic DNA was extracted from peripheral blood samples collected at baseline using standard methods. A total of 188 patients of the ParkWest cohort were characterized by whole exome sequencing and 5 nonsynonymous variants were detected (N370S, T369M, E326K, V460L, and Y135C) and confirmed by sequencing. ${ }^{5}$ These variants were genotyped in all available samples using TaqMan single nucleotide polymorphism genotyping assay (Thermo Fisher Scientific). The L444P genotype was 
determined using PCR-restriction fragment length polymorphism assays (primer sequences and reaction conditions available on request) and all mutations confirmed by direct sequencing of the PCR- product. ${ }^{19,20}$

All amino acid substitutions are numbered excluding the 39residue signal peptide.

\section{Statistical Methods}

"GBA carriers" included all patients carrying any of the detected nonsynonymous GBA variants. We further split GBA carriers into "polymorphism carriers" (E326K, T369M, or V460L) and "deleterious carriers" (Y135C, N370S, or L444P) as described. ${ }^{5}$ We compared between-group differences at baseline using $t$ tests, Mann-Whitney $U$ tests, and $\chi^{2}$ tests as appropriate.

We applied mixed linear regression analysis to determine the association between GBA genotype and longitudinal motor measures assessed using repeat UPDRS ADL and motor scores. Mixed models use all available data during follow-up, can handle missing at random data, and can properly account for correlation between repeated measurements: at each visit between $94.8 \%$ and $99.8 \%$ of patients had complete UPDRS $\mathrm{ADL}$ or motor score data. We performed the analyses without adjustment and with adjustment for sex, study cohort, age, and duration of motor symptoms at baseline. The effect sizes were comparable after additional adjustment for LED at each visit (data not shown). The time of the repeated measures was computed in years from the baseline date. All models have fixed effect of time, as well as random intercepts, random effect of time, and first-order autoregressive residual covariance structure. Tremor and axial impairment scores were log-transformed before analysis. Plot of predictive margins was created in Stata using margins and marginsplot. As estimates of associations may be affected by dropouts due to death, ${ }^{21}$ we performed post hoc sensitivity analysis by joint modeling of longitudinal measures of motor function and time to death using function stjm in Stata. ${ }^{22}$ Whereas the longitudinal measures of UPDRS ADL or motor score and the event times were found to be related, the estimated associations and effect sizes between GBA carrier status and the longitudinal measures were very similar to the presented results (data not shown).

Statistical analyses were conducted using IBM SPSS Statistics (Armonk, NY) version 26.0 or Stata and 2-tailed $p$ values $<0.05$ considered significant.

\section{Power Calculations for the Clinical Trial}

We performed power calculations for a hypothetical clinical trial of a putative neuroprotective agent with 36 months follow-up. The first scenario assumed that all newly diagnosed patients with PD ("all-comers") would be eligible for the trial, and the second limited trial selection to carriers of a GBA variant. The expected trajectories for the placebo group were set to those estimated in the present study from population- averaged models adjusted for age, sex, cohort, and disease duration (for all patients or only GBA carriers, as appropriate). The covariance matrices were based on separate estimates of both variance and correlation, that is, respectively 137 and 0.77 for all comers and 102 and 0.67 for GBA carriers. The treated individuals were assumed to experience a $50 \%$ reduction in their motor decline (i.e., the intervention halves disease progression). The required sample sizes to obtain a power of between $60 \%$ and $96 \%$ to detect such differences in slopes (i.e., the between-within subjects interaction effect) at a $5 \%$ significance level were estimated using Stata function power repeated, which incorporates $\mathrm{F}$ tests with GreenhouseGeisser correction for lack of sphericity.

\section{Data Availability}

Anonymized data are available on request by any qualified investigator for purposes of replicating procedures and results.

\section{Results}

\section{Baseline Profile of PD-GBA Carriers}

A total of 440 patients with PD were included in the study. Their mean age at baseline was $69.9( \pm 9.6)$ years, with $60.7 \%$ (267) male (table 1). The median duration of followup was 7.0 years (interquartile range 2.0). During the study, 117 patients died (26.6\%) and $25(5.7 \%)$ withdrew from the study (figure 1). GBA variants were identified in 53 patients: 29 E326K, 16 T369M, 6 L444P, 1 Y135C, 1 N370S, and 1 V460L variant. ${ }^{5}$ Carriers of any GBA variant observed the first motor signs of PD at an earlier age than noncarriers $(p=0.022)$ and were also younger at the age of PD diagnosis $(p=0.014)$, as previously reported. ${ }^{5}$ However, GBA carriers and noncarriers did not differ at the time of diagnosis in terms of overall motor severity or ADL function, nor severity of tremor, rigidity, bradykinesia, or axial impairment (all $p>0.1$ ).

\section{Effect of GBA on Motor and Functional Decline}

Linear mixed models with adjustment for age, sex, study cohort, and duration of motor symptoms at baseline demonstrated a $69 \%$ more rapid motor decline per year in GBA carriers than noncarriers, with an annual increase in UPDRS motor score of 2.2 (95\% CI 1.3-3.1 points) vs 1.3 point (95\% CI 1.1-1.6 point), respectively. This difference was significant (0.9 point difference per year; 95\% CI 0.2-1.5; $p=0.007$ ) (table 2 and figure 2). We observed a similar effect on the rate of functional decline, with carriers of a GBA variant predicted to exhibit an annual increase in UPDRS ADL score of 1.5 point (95\% CI 1.1 to 2.0 ), compared to only 1.0 point (95\% CI $0.9-1.1$ point) in noncarriers (0.5 point difference per year; 95\% CI $0.2-0.9 ; p=0.003$ ). The effect sizes were comparable for adjusted and unadjusted models (data not shown).

Both the polymorphism and the deleterious mutation carrier groups showed faster motor and functional decline compared 
Table 1 Demographic and Clinical Measures at the Time of Parkinson Disease (PD) Diagnosis

\begin{tabular}{llll}
\hline & $\begin{array}{l}\text { All patients } \\
\text { with PD }\end{array}$ & Noncarriers & GBA carriers \\
\hline Total & 440 & 387 & 53 \\
\hline Male, $\mathbf{n}(\%)$ & $267(60.7)$ & $233(60.2)$ & $34(64.2)$ \\
\hline $\begin{array}{l}\text { Age at baseline, } \\
\mathbf{y}, \text { mean ( } \pm \text { SD) }\end{array}$ & $69.9(9.6)$ & $70.3(9.5)$ & $66.8(10.1)$ \\
\hline $\begin{array}{l}\text { Age at motor onset, } \\
\text { y, mean ( } \pm \text { SD) }\end{array}$ & $67.8(9.7)$ & $68.1(9.6)$ & $65.0(9.8)$ \\
\hline $\begin{array}{l}\text { Family history } \\
\text { Years of education }\end{array}$ & $111.4(3.4)$ & $11.4(3.4)$ & $11.6(5.0)$ \\
\hline MMSE, median (IQR) & $29.0(3.0)$ & $29.0(3.0)$ & $28.3(2.5)$ \\
\hline H\&Y, median (IQR) & $2.0(1.0)$ & $2.0(1.0)$ & $2.0(1.0)$ \\
\hline UPDRS ADL (IQR) & $9.0(6.0)$ & $9.0(6.0)$ & $8.0(7.0)$ \\
\hline UPDRS motor (IQR) & $23.0(16.0)$ & $23.0(17.0)$ & $21.0(15.5)$ \\
\hline
\end{tabular}

UPDRS motor subscores,

median (IQR)

\begin{tabular}{llll}
\hline Tremor & $3.0(4.0)$ & $3.0(4.0)$ & $3.0(4.0)$ \\
\hline Bradykinesia & $11.0(8.0)$ & $11.0(8.25)$ & $10.0(7.5)$ \\
\hline Rigidity & $4.0(5.0)$ & $4.0(4.0)$ & $4.0(5.0)$ \\
\hline Axial impairment & $2.0(2.0)$ & $2.0(3.0)$ & $2.0(2.0)$ \\
\hline
\end{tabular}

Abbreviations: $A D L=$ activities of daily living; $H \& Y=$ Hoehn \& Yahr score; IQR = interquartile range; MMSE = Mini-Mental State Examination; UPDRS = Unified Parkinson's Disease Rating Scale.

to noncarriers (table 2). The predicted difference in change in annual UPDRS ADL score compared to noncarriers was highest for the deleterious mutation carrier group ( 0.9 point difference per year; 95\% CI 0.1-1.7; $p=0.029)$ and more modest for the polymorphism carrier group ( 0.4 point difference per year; 95\% CI $0.1-0.8 ; p=0.016)$. The same tendency was observed for change in UPDRS motor score per year, with a more pronounced motor decline predicted for carriers of a GBA mutation (1.2 point difference per year; $95 \%$ CI -0.3 to $2.7 ; p=0.11)$ than a polymorphism (0.8 point difference per year; 95\% CI $0.1-1.5 ; p=0.022$ ).

Examination of the change in specific motor symptoms revealed that the difference in motor decline was mainly driven by changes in the scores for bradykinesia and rigidity. Bradykinesia score was predicted to increase by 0.5 point per year more in carriers of a GBA variant compared to noncarriers (95\% CI $0.2-0.8 ; p=0.002)$. Further, the annual change in the bradykinesia subscore was significantly larger in carriers of a GBA mutation than in carriers of a GBA polymorphism when compared to noncarriers (table 3 ). Rigidity score also increased faster when comparing GBA carriers to noncarriers (0.2 point difference per year; $95 \%$ CI $0.0-0.3 ; p=0.038$ ), but no significant differences were observed for rigidity when comparing either GBA mutation or polymorphism carriers to noncarriers (table 3 ).

\section{Hypothetical Power Analysis for a Stratified Clinical Trial Targeting GBA-PD}

Enriching a clinical trial for subgroups of patients predicted to progress faster in the primary outcome can considerably reduce trial size. To assess the reductions in trial size that could be achieved by recruiting only carriers of GBA variants compared to an all-comer design, we ran clinical trial power analysis for a hypothetical drug predicted to halve the progression of motor impairment (measured using UPDRS motor score). In a 3 -year trial designed to have $80 \%$ power to detect between-within subjects interaction effect, we found that the required size of the trial was reduced from 1,446 participants if all newly diagnosed patients were eligible to 506 in the GBA-carrier design, providing a $65 \%$ reduction in the number of individuals that need to be enrolled (figure 3).

Figure 1 Flowchart of Study Participants

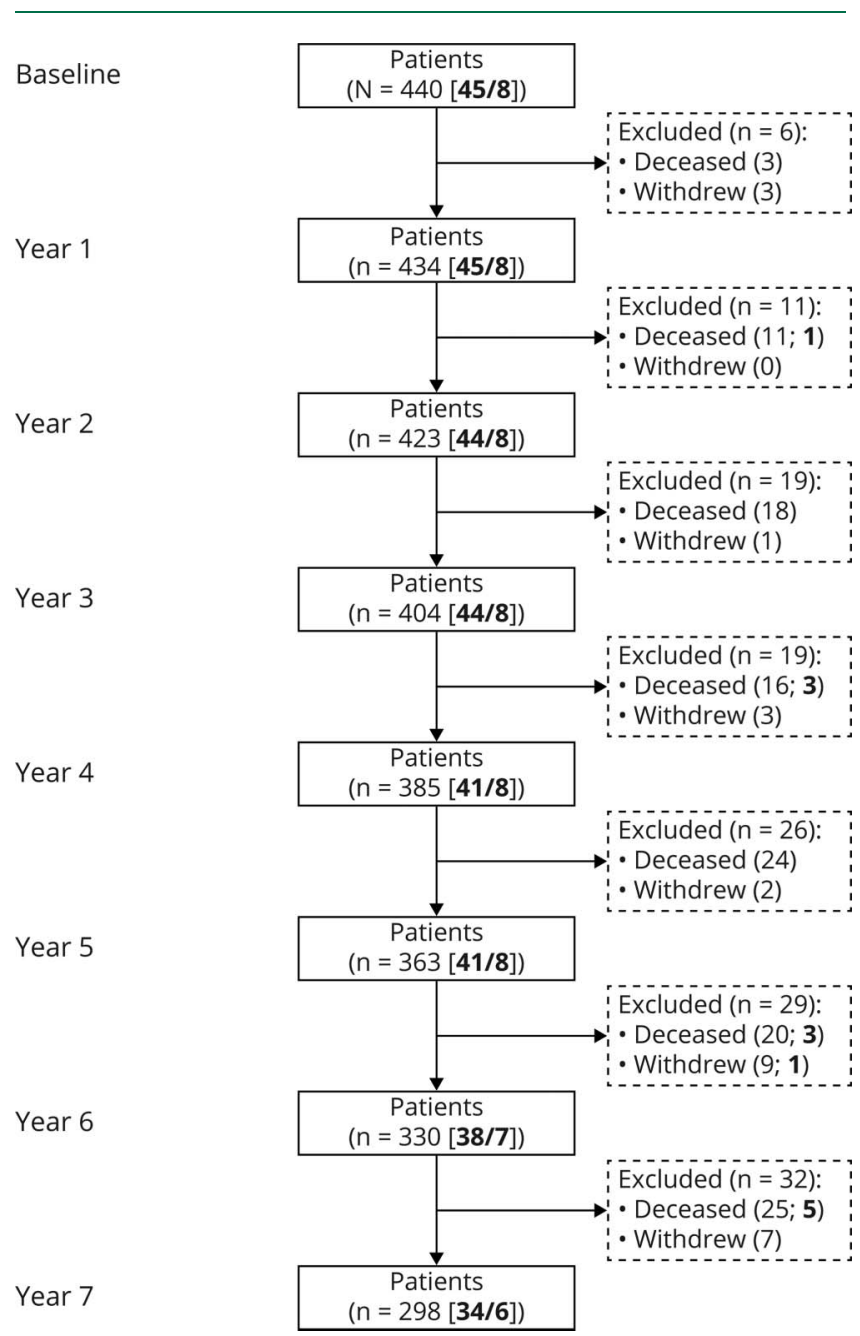

Overview of patient inclusion from baseline until the 7-year visit. The number of patients in the study at each visit is shown. Withdrawals and deaths between visits are shown in dashed boxes. The number of patients carrying a GBA polymorphism/mutation is shown in bold. The flowchart is simplified for readability. 
Table 2 Relationship Between GBA Carrier Status and Change in Unified Parkinson's Disease Rating Scale (UPDRS) Motor and Activities of Daily Living (ADL) Scores Using Linear Mixed Effects Models

\begin{tabular}{|c|c|c|c|c|c|c|}
\hline \multirow[b]{2}{*}{ Subscale } & \multicolumn{2}{|c|}{ GBA carrier $(n=53)^{a}$} & \multicolumn{2}{|c|}{ Mutation carrier $(n=8)^{a}$} & \multicolumn{2}{|c|}{ Polymorphism carrier $(n=45)^{a}$} \\
\hline & $\beta(95 \% \mathrm{Cl})$ & $p$ Value & $\beta(95 \% \mathrm{Cl})$ & $p$ Value & $\beta(95 \% \mathrm{Cl})$ & $p$ Value \\
\hline \multicolumn{7}{|l|}{ UPDRS motor } \\
\hline Main effect ${ }^{\mathbf{b}}$ & $-0.3(-3.1$ to 2.4$)$ & 0.80 & $1.2(-5.5$ to 7.9$)$ & 0.72 & $-0.6(-3.5$ to 2.3$)$ & 0.69 \\
\hline Interaction with time ${ }^{b}$ & $0.9(0.2$ to 1.5$)$ & 0.007 & $1.2(-0.3$ to 2.7$)$ & 0.11 & 0.8 (0.1 to 1.5$)$ & 0.022 \\
\hline \multicolumn{7}{|l|}{ UPDRS ADL } \\
\hline Main effect ${ }^{\mathbf{b}}$ & $0.1(-1.2$ to 1.3$)$ & 0.92 & $1.4(-1.7$ to 4.5$)$ & 0.37 & $-0.2(-1.5$ to 1.2$)$ & 0.82 \\
\hline Interaction with time ${ }^{b}$ & $0.5(0.2$ to 0.9$)$ & 0.003 & $0.9(0.1$ to 1.7$)$ & 0.029 & $0.4(0.1$ to 0.8$)$ & 0.016 \\
\hline
\end{tabular}

Abbreviation: $\mathrm{Cl}=$ confidence interval.

${ }^{a}$ GBA carrier status compared to noncarriers: UPDRS motor scores were available for 386 noncarriers and 53 GBA carriers and UPDRS ADL scores were available for 382 noncarriers and 53 GBA carriers.

${ }^{\mathrm{b}}$ Models adjusted for sex, study cohort, and age and duration of motor symptoms at baseline. The main effect indicates the effect of carrier status on the intercept and the interaction with time indicates the effect of carrier status on the slope (change in value per year) of the model.

\section{Discussion}

In this study, we comprehensively explored the relationship between GBA carrier status and long-term motor and functional decline in newly diagnosed patients with PD followed from time of diagnosis. Taking advantage of longitudinal data from 3 population-based studies of patients with PD in Northern Europe, our findings support a strong association between GBA carrier status and faster progression in motor symptoms, measured using widely used clinical and patientreported measures of motor impairment. These data have important implications for counseling patients regarding disease prognosis and for planning and interpreting clinical trials of disease-modifying therapies in PD.

GBA-PD has long been associated with a younger onset of PD and a more malignant disease course, with increased dementia risk and mortality, ${ }^{3,6-11}$ but studies evaluating the progression of motor impairment using the UPDRS, particularly in early PD, have been surprisingly few and inconsistent. Only 2 other studies have previously shown an association of GBA variants and motor impairment measured by the UPDRS, and both of these did so only in patients with more advanced disease. The first followed 13 patients with PD (average disease duration 7.5 years) and the 2 most common pathogenic GBA mutations (N370S or L444P) and 26 matched noncarriers, and showed more rapid motor progression in patients with GBA-PD. ${ }^{8}$ Subsequently, a large multicenter study of 733 patients (average disease duration 8.6 years) showed that carriers of a GBA variant experienced a larger increase in UPDRS motor score compared to noncarriers. ${ }^{9}$ Another study looked at the association of 2 individual GBA variants (N370S or $\mathrm{K} 26 \mathrm{R} / \mathrm{rs} 75548401$ ) selected from the PDgene database and progression of UPDRS motor score. No significant association was shown between either variant and the change in UPDRS motor score measured in participants from the Washington
University cohort and Parkinson's Progression Markers Initiative (PPMI) cohorts using UPDRS scores in the "on" or "off" state. ${ }^{23}$ Of note, the low observed minor allele frequency of each variant (N370S 0.007 and K26R 0.012) combined with moderate sample size and length of follow-up of the cohorts may account for the lack of significant associations. The analysis of individual GBA variants may require larger collaborations; indeed the largest genome-wide association study of PD clinical phenotypes to date included 12 cohorts (including PPMI) and whereas no significant associations were identified with UPDRS motor scores, GBA T369M was shown to be associated with the faster development of $\mathrm{H} \& \mathrm{Y}$ stage $3 .^{24}$ Alternatively, a more comprehensive assessment of $G B A$ variants using exome sequencing, as done in the present

Figure 2 Prediction of Unified Parkinson's Disease Rating Scale (UPDRS) Motor Score Over Time

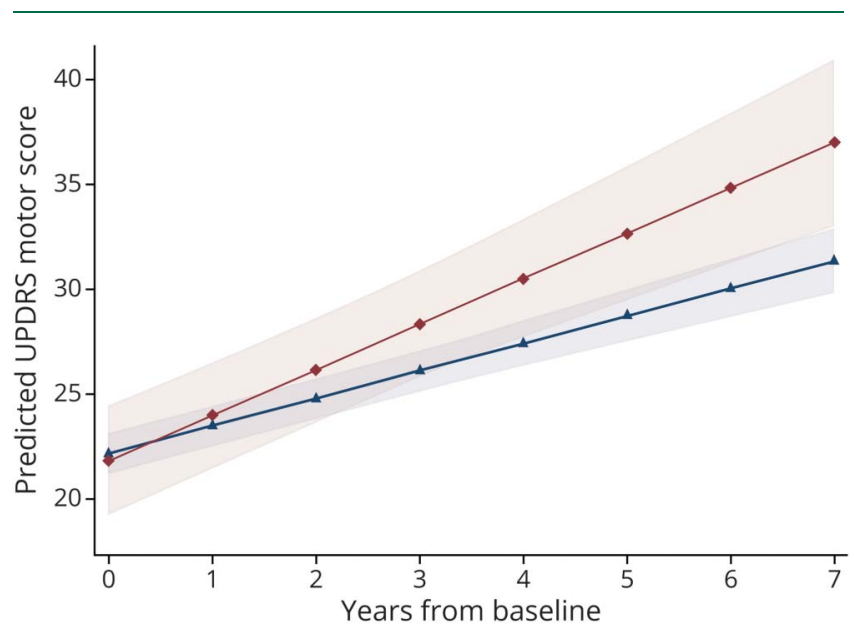

Average predicted UPDRS motor scores with confidence bands for the first 7 years after diagnosis of PD for carriers of GBA variants (red, diamonds) and noncarriers (blue, triangles). 
Table 3 Relationship Between GBA Carrier Status and Change in Unified Parkinson's Disease Rating Scale (UPDRS) Motor Subscores Using Linear Mixed Effects Model

\begin{tabular}{|c|c|c|c|c|c|c|}
\hline \multirow[b]{2}{*}{ Subscale } & \multicolumn{2}{|l|}{ GBA carrier $(n=53)^{a}$} & \multicolumn{2}{|c|}{ Mutation carrier $(n=8)^{a}$} & \multicolumn{2}{|c|}{ Polymorphism carrier $(n=45)^{a}$} \\
\hline & $\beta(95 \% \mathrm{Cl})$ & $p$ Value & $\beta(95 \% \mathrm{Cl})$ & $p$ Value & $\beta(95 \% \mathrm{Cl})$ & $p$ Value \\
\hline \multicolumn{7}{|l|}{ Tremor $^{\mathbf{b}}$} \\
\hline Main effect ${ }^{c}$ & $0.05(-0.2$ to 0.2$)$ & 0.85 & $0.3(-0.2$ to 0.7$)$ & 0.21 & $-0.0(-0.2$ to 0.2$)$ & 0.76 \\
\hline Interaction with time ${ }^{c}$ & $0.02(-0.01$ to 0.05$)$ & 0.21 & $-0.04(-0.12$ to 0.04$)$ & 0.31 & $0.03(-0.00$ to 0.07$)$ & 0.074 \\
\hline \multicolumn{7}{|l|}{ Bradykinesia } \\
\hline Main effect ${ }^{c}$ & $-0.6(-2.0$ to 0.8$)$ & 0.39 & $-0.3(-3.7$ to 3.2$)$ & 0.88 & $-0.7(-2.2$ to 0.8$)$ & 0.38 \\
\hline Interaction with time ${ }^{c}$ & 0.49 (0.18 to 0.79$)$ & 0.002 & 0.85 (0.13 to 1.58$)$ & 0.021 & 0.41 (0.08 to 0.75$)$ & 0.014 \\
\hline \multicolumn{7}{|l|}{ Rigidity } \\
\hline Main effect ${ }^{c}$ & $0.4(-0.4$ to 1.2$)$ & 0.32 & $-0.1(-2.1$ to 1.9$)$ & 0.92 & $0.5(-0.4$ to 1.4$)$ & 0.25 \\
\hline Interaction with time ${ }^{c}$ & 0.17 (0.01 to 0.32$)$ & 0.038 & $0.32(-0.05$ to 0.69$)$ & 0.089 & $0.14(-0.03$ to 0.31$)$ & 0.12 \\
\hline \multicolumn{7}{|l|}{ Axial impairment } \\
\hline Main effect ${ }^{c}$ & $-0.1(-0.7$ to 0.6$)$ & 0.87 & $0.0(-1.6$ to 1.6$)$ & 1.00 & $-0.1(-0.8$ to 0.6$)$ & 0.86 \\
\hline Interaction with time ${ }^{c}$ & $0.10(-0.07$ to 0.27$)$ & 0.27 & $0.09(-0.32$ to 0.50$)$ & 0.66 & $0.10(-0.09$ to 0.29$)$ & 0.30 \\
\hline
\end{tabular}

Abbreviation: $\mathrm{Cl}=$ confidence interval.

a GBA carrier status compared to noncarriers: subscores were available for 386 noncarriers and 53 GBA carriers.

b The tremor and axial impairment scores were log-transformed before analysis.

${ }^{c}$ Models adjusted for sex, study cohort, and age and duration of motor symptoms at baseline. The main effect indicates the effect of carrier status on the intercept and the interaction with time indicates the effect of carrier status on the slope (change in value per year) of the model.

study, could determine whether GBA carrier status affects motor impairment in these cohorts.

In our population-based study, we followed patients prospectively from the time of PD diagnosis with regular and structured clinical follow-up for up to 7 years and found carriers of a $G B A$ variant on average experience a more than $69 \%$ greater annual increase in UPDRS motor score compared to noncarriers. Over the course of the study, the model predicts carriers of a GBA variant will experience on average a 7-point higher increase in UPDRS motor score compared to the noncarriers. This is considered to represent a moderate to large clinically important difference in motor function. ${ }^{25}$ In support of this, we also show substantial differences in the rate of functional decline measured by the ADL scores. Our data add an important missing piece of the puzzle, showing for the first time that carriers of $G B A$ variants experience a more aggressive motor disease course from the early phases of PD.

Because GBA variants were associated with more rapid progression of overall motor symptoms, we further examined whether and to what extent GBA carrier status was associated with changes in the severity of specific motor signs. Significant differences between groups were shown in progression rates in the subscores related to bradykinesia and rigidity. It is well known that patients with PD are prone to develop dementia, with axial impairment being an established clinical risk factor. ${ }^{26}$ However, we found no association between GBA carrier status and change in severity of axial symptoms in our study. In contrast, we observed clear associations with worsening of bradykinesia and rigidity, which also have been associated

Figure 3 Reduced Trial Size in GBA-Targeted Clinical Trials Compared to the Traditional "All-Comer" Design

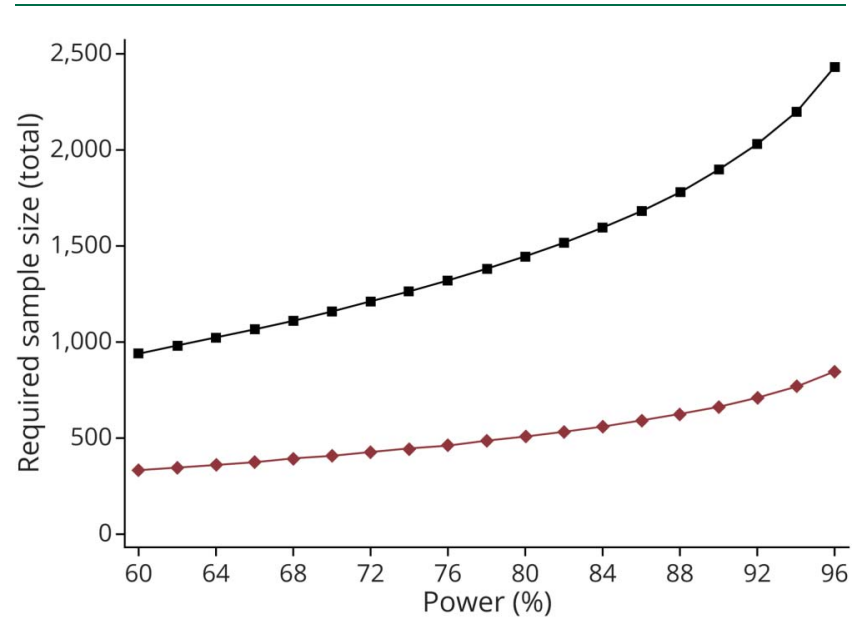

Required sample size for clinical trials enrolling only carriers of GBA variants (red diamonds) or an "all-comer" design with nonselected patients with Parkinson disease (PD) (black squares) across varying levels of power to detect a between-within subjects interaction effect. A trial recruiting only PD carriers of a GBA variant could reduce the size required by threefold for an intervention indicated to halve the rate of motor decline, measured by the Unified Parkinson's Disease Rating Scale (UPDRS) motor score. 
with an increased dementia risk. Interestingly, while advanced age is considered a major risk factor for PD dementia, ${ }^{27,28}$ we and others have previously shown that GBA carriers develop $\mathrm{PD}$ at younger age than noncarriers. Overall, our data suggest that GBA is associated with a specific subtype of PD with more rapid motor progression and higher dementia risk despite lower age at onset and only milder axial symptoms.

We observed that the effect on changes in both the ADL and motor examination scores was faster in carriers of a GBA mutation compared to carriers of a GBA polymorphism. Similar results were observed by Davis et al., ${ }^{9}$ who reported that the effect on UPDRS motor score was slightly more pronounced in carriers of pathogenic GBA mutations compared to carriers of the common E326K polymorphism. This observation is also in line with previous work showing that the effect size associated with GBA carrier status on cognitive impairment is also on a continuum. ${ }^{3,9-11}$ Our findings reaffirm the importance of the severity of the GBA variant on the progression of disease in PD.

It is established that GBA carriers have an earlier age at PD onset but there are conflicting reports of the presenting symptoms in GBA-PD compared to idiopathic forms of the disease. ${ }^{29-32}$ In our study, patients with PD-associated GBA mutations appeared clinically indistinguishable from newly diagnosed patients with idiopathic PD. This is in agreement with the only other population-based study to assess GBA carriers at the time of PD diagnosis, which found that patients with GBA-PD had a similar motor profile compared to noncarriers. ${ }^{3}$ Although we would not yet advocate for routine genetic testing, these data suggest that screening for GBA variants might be helpful as part of a model to identify those at risk of a more malignant disease course at diagnosis.

The UPDRS motor score is one of the most common outcomes in observational studies and clinical trials, used to measure the severity of PD impairment. The advantages as a primary outcome include good intrarater and inter-rater reliability but, as has been shown in this study, genetic heterogeneity in populations can substantially influence the change in UPDRS motor scores over time. We show that the GBA-PD subgroup, which constitutes about $12 \%$ of the PD population, are predicted to show on average $69 \%$ more rapid motor progression than the noncarriers. If not accounted for, differences in GBA carrier distribution by treatment arm (which can arise by chance even during randomization) could thus influence trial outcome and lead to invalid conclusions. Balancing treatment arms with stratification of randomization according to GBA status would avoid this potential problem in future trials.

A major challenge for drug development in neurodegenerative diseases is that adequately powered trials typically require several hundred participants and long durations. The expected progression of the trial's primary outcome in genetic subgroups can be used to improve the efficiency and effectiveness of clinical trials. The utility of this model in GBA-PD was assessed by comparing simulated randomized placebo- controlled trial designs to recruit all newly diagnosed patients with PD (all-comers) or only those predicted to be at risk of a faster motor decline, by limiting trial selection to carriers of a $G B A$ variant. We show that a trial limited to GBA carriers could recruit up to $65 \%$ fewer participants than a traditional all-comer design, which could feasibly translate into considerable savings in costs. Our data are in line with and extend on previous estimations targeting only patients with a GBA mutation (such as L444P) for a hypothetical trial with MMSE as the primary outcome. ${ }^{11}$ Notably, this hypothetical trial was found to reduce trial size by as much as 25 -fold compared to a trial of patients with PD without a GBA mutation. ${ }^{11}$ However, neuropathic GBA mutations are rare in the general PD population ( $1.4 \%$ in our study), whereas we considered all types of GBA variant and thus substantially increased the number of eligible trial participants. In light of this, the threefold reduction in trial size shown here is more realistic in a population-based setting. The first example of a trial studying ambroxol in genetically defined patients with PD was recently published, ${ }^{33}$ and although this trial was not powered to assess the clinical efficacy of the drug, it demonstrates the potential of using genetic information to balance or build a trial.

Our study is not without limitations. The modest sample size of each genotype group prevented the assessment of individual GBA mutations and larger longitudinal studies will be needed to determine whether the risk for motor progression varies across individual GBA variants. The use of a linear model provides an approximation of overall disease progression, as patients tend to improve during the first year after initiating medication before worsening again, and future studies should also examine possible differences in the response to medication in GBA-associated PD. Strengths of our study include the large number of participants with newly diagnosed PD with longitudinal data available for inclusion, the uniform study designs of the 3 cohorts, length of follow-up (median 7 years), the number of visits analyzed per participant (median 8 visits), and the exceptionally low attrition rate. Furthermore, each of the cohorts are population-based studies, which avoids some of the pitfalls when cases are recruited from neurology and memory clinics. This means that our findings that GBA variants are linked to more rapid motor progression in PD are likely to be applicable to the general PD population. Motor decline has a major bearing on independence, nursing home admission, caregiver burden, and mortality in $\mathrm{PD},{ }^{34}$ and better understanding and prediction of PD progression could improve disease management for a meaningful proportion of patients and has direct implications for improving the design of clinical trials.

\section{Acknowledgment}

The authors thank the patients for participating in the studies; ParkWest thanks all members of the Norwegian ParkWest study group and other personnel involved in the study; NYPUM thanks the study nurse/coordinator Mona Edström and laboratory technician Jörgen Andersson for database management; and PINE thanks the research fellows (Kate 
Taylor, Robert Caslake, and David McGhee) and study nurses (Clare Harris, Joanna Gordon, Anne Hayman, and Hazel Forbes) who assessed participants, the secretaries (Susan Kilpatrick and Pam Rebecca), the data management team (Katie Wilde and David Ritchie), and the clinicians who referred patients to the PINE study.

\section{Study Funding}

The Norwegian ParkWest study has been funded by the Research Council of Norway (grant 177966) and the Western Norway Regional Health Authority (grant 911218) and the Norwegian Parkinsons Disease Association. J.M.-G. and G.A. are supported by the Research Council of Norway (grant 287842). PINE was supported by Parkinson's UK (grants G0502, G0914, and G1302), Scottish Government Chief Scientist Office, BMA Doris Hillier Award, the BUPA Foundation, NHS Grampian Endowments, and RS MacDonald Trust. The NYPUM study has been funded by the Swedish Medical Research Council, the Swedish Parkinson's Disease Association, the Swedish Parkinson Foundation, and the Västerbotten County Council.

\section{Disclosure}

J. Maple-Grødem, I. Dalen, and O.-B. Tysnes report no disclosures relevant to the manuscript. A. Macleod reports fellowship funding from the Scottish Chief Scientist Office (PCL/17/10) and grant funding from the Academy of Medical Sciences and NHS Grampian Endowments. L. Forsgren reports no disclosures relevant to the manuscript. C. Counsell reports NHS Grampian Endowments and RS Macdonald Trust. G. Alves reports Norwegian Research Council grant 287842, principal investigator, 2019-2022 and support from University of Stavanger, Norwegian Parkinson Research Foundation, and Reberg's Legacy. Go to Neurology. org $/ \mathrm{N}$ for full disclosures.

\section{Publication History}

Received by Neurology May 21, 2020. Accepted in final form October 14, 2020.

\section{Appendix Authors}

\begin{tabular}{lll}
\hline Name & Location & Contribution \\
\hline Jodi Maple- & Stavanger & $\begin{array}{l}\text { Study concept or design; analysis } \\
\text { Grødem, PhD }\end{array}$ \\
$\begin{array}{l}\text { University } \\
\text { Hospital }\end{array}$ & $\begin{array}{l}\text { drafting/revision of the manuscript } \\
\text { for intellectual content, including } \\
\text { medical writing for content }\end{array}$ \\
\end{tabular}

\begin{tabular}{ll}
\hline Ingvild Dalen, & Stavanger \\
PhD & University \\
& Hospital
\end{tabular}

Study concept or design; analysis or interpretation of the data; drafting/revision of the manuscript for intellectual content, including medical writing for content

\begin{tabular}{|c|c|c|}
\hline $\begin{array}{l}\text { Ole-Bjørn } \\
\text { Tysnes, MD, } \\
\text { PhD }\end{array}$ & $\begin{array}{l}\text { Haukeland } \\
\text { University } \\
\text { Hospital, Bergen }\end{array}$ & $\begin{array}{l}\text { Major role in the acquisition of } \\
\text { data; drafting/revision of the } \\
\text { manuscript for intellectual } \\
\text { content, including medical writing } \\
\text { for content }\end{array}$ \\
\hline
\end{tabular}

\section{Appendix (continued)}

\begin{tabular}{lll}
\hline $\begin{array}{l}\text { Angus D. } \\
\text { Macleod, }\end{array}$ & University of & Major role in the acquisition of \\
MRCP, PhD & data; analysis or interpretation of \\
& & $\begin{array}{l}\text { the data; drafting/revision of the } \\
\text { manuscript for intellectual } \\
\text { content, including medical writing } \\
\text { for content }\end{array}$
\end{tabular}

\begin{tabular}{|c|c|c|}
\hline $\begin{array}{l}\text { Lars } \\
\text { Forsgren, MD, } \\
\text { PhD }\end{array}$ & Umeå University & $\begin{array}{l}\text { Major role in the acquisition of } \\
\text { data; analysis or interpretation of } \\
\text { the data; drafting/revision of the } \\
\text { manuscript for content, including } \\
\text { medical writing for content }\end{array}$ \\
\hline
\end{tabular}

\begin{tabular}{lll}
\hline Carl E. & University of & Major role in the acquisition of \\
Counsell, & Aberdeen & data; study concept or design; \\
analysis or interpretation of the \\
data; drafting/revision of the \\
manuscript for content, including \\
medical writing for content
\end{tabular}

\begin{tabular}{lll}
\hline Guido Alves, & Stavanger & Study concept or design; major \\
MD, PhD & University & role in the acquisition of data; \\
& analysis or interpretation of the \\
data; drafting/revision of the \\
manuscript for content, including \\
\end{tabular}

\section{References}

1. Sidransky E, Nalls MA, Aasly JO, et al. Multicenter analysis of glucocerebrosidase mutations in Parkinson's disease. N Engl J Med 2009;361:1651-1661.

2. Sidransky E, Lopez G. The link between the GBA gene and parkinsonism. Lancet Neurol 2012;11:986-998.

3. Winder-Rhodes SE, Evans JR, Ban M, et al. Glucocerebrosidase mutations influence the natural history of Parkinson's disease in a community-based incident cohort. Brain 2013;136:392-399.

4. Jesus S, Huertas I, Bernal-Bernal I, et al. GBA variants influence motor and non-motor features of Parkinson's disease. PLoS One 2016;11:e0167749.

5. Lunde KA, Chung J, Dalen I, et al. Association of glucocerebrosidase polymorphisms and mutations with dementia in incident Parkinson's disease. Alzheimers Dement 2018;14:1293-1301.

6. Gan-Or Z, Giladi N, Rozovski U, et al. Genotype-phenotype correlations between GBA mutations and Parkinson disease risk and onset. Neurology 2008;70: 2277-2283.

7. Clark LN, Ross BM, Wang Y, et al. Mutations in the glucocerebrosidase gene are associated with early-onset Parkinson disease. Neurology 2007;69:1270-1277.

8. Brockmann K, Srulijes K, Pflederer S, et al. GBA-associated Parkinson's disease: reduced survival and more rapid progression in a prospective longitudinal study. Mov Disord 2015;30:407-411.

9. Davis MY, Johnson CO, Leverenz JB, et al. Association of GBA mutations and the E326K polymorphism with motor and cognitive progression in Parkinson disease. JAMA Neurol 2016;73:1217-1224.

10. Cilia R, Tunesi S, Marotta G, et al. Survival and dementia in GBA-associated Parkinson's disease: the mutation matters. Ann Neurol 2016;80:662-673.

11. Liu G, Boot B, Locascio JJ, et al. Specifically neuropathic Gaucher's mutations accelerate cognitive decline in Parkinson's. Ann Neurol 2016;80:674-685.

12. Alves G, Muller B, Herlofson K, et al. Incidence of Parkinson's disease in Norway: the Norwegian ParkWest study. J Neurol Neurosurg Psychiatry 2009;80:851-857.

13. Linder J, Stenlund H, Forsgren L. Incidence of Parkinson's disease and parkinsonism in northern Sweden: a population-based study. Mov Disord 2010;25:341-348.

14. Caslake R, Taylor K, Scott N, et al. Age-, gender-, and socioeconomic status-specific incidence of Parkinson's disease and parkinsonism in northeast Scotland: the PINE study. Parkinsonism Relat Disord 2013;19:515-521.

15. Backstrom D, Eriksson Domellof M, Granasen G, et al. Polymorphisms in dopamineassociated genes and cognitive decline in Parkinson's disease. Acta Neurol Scand 2018;137:91-98.

16. Goetz CG, Poewe W, Rascol O, et al. Movement disorder Society Task Force report on the Hoehn and Yahr staging scale: status and recommendations. Mov Disord 2004; 19:1020-1028.

17. Fahn S, Elton R. Unified Parkinson's Disease Rating Scale. In: Fahn S, Marsden CD, Calne D, Goldstein M, eds. Recent Developments in Parkinson's Disease. Florham Park, NJ: Macmillan Healthcare Information; 1987;1987:153-163.

18. Tomlinson CL, Stowe R, Patel S, Rick C, Gray R, Clarke CE. Systematic review of levodopa dose equivalency reporting in Parkinson's disease. Mov Disord 2010;25: 2649-2653.

19. Stone DL, Tayebi N, Orvisky E, Stubblefield B, Madike V, Sidransky E. Glucocerebrosidase gene mutations in patients with type 2 Gaucher disease. Hum Mutat 2000; 15:181-188. 
20. Torok R, Zadori D, Torok N, Csility E, Vecsei L, Klivenyi P. An assessment of the frequency of mutations in the GBA and VPS35 genes in Hungarian patients with sporadic Parkinson's disease. Neurosci Lett 2016;610:135-138.

21. Raitanen J, Stenholm S, Tiainen K, Jylha M, Nevalainen J. Longitudinal change in physical functioning and dropout due to death among the oldest old: a comparison of three methods of analysis. Eur J Ageing 2020;17:207-216.

22. Crowther MJ, Abrams KR, Lambert PC. Joint modeling of longitudinal and survival data. Stata J 2013;13:165-184.

23. Davis AA, Andruska KM, Benitez BA, Racette BA, Perlmutter JS, Cruchaga C. Variants in GBA, SNCA, and MAPT influence Parkinson disease risk, age at onset, and progression. Neurobiol Aging 2016;37:209.e201-209.e207.

24. Iwaki H, Blauwendraat C, Leonard HL, et al. Genomewide association study of Parkinson's disease clinical biomarkers in 12 longitudinal patients' cohorts. Mov Disord 2019;34:1839-1850.

25. Shulman LM, Gruber-Baldini AL, Anderson KE, Fishman PS, Reich SG, Weiner WJ. The clinically important difference on the Unified Parkinson's Disease Rating Scale. Arch Neurol 2010;67:64-70.

26. Burn DJ, Rowan EN, Allan LM, Molloy S, O’Brien JT, McKeith IG. Motor subtype and cognitive decline in Parkinson's disease, Parkinson's disease with dementia, and dementia with Lewy bodies. J Neurol Neurosurg Psychiatry 2006; $77: 585-589$
27. Wickremaratchi MM, Ben-Shlomo Y, Morris HR. The effect of onset age on the clinical features of Parkinson's disease. Eur J Neurol 2009;16:450-456.

28. Kempster PA, O'Sullivan SS, Holton JL, Revesz T, Lees AJ. Relationships between age and late progression of Parkinson's disease: a clinico-pathological study. Brain 2010;133:1755-1762.

29. Zhang Y, Sun QY, Zhao YW, et al. Effect of GBA mutations on phenotype of Parkinson's disease: a study on Chinese population and a meta-analysis. Parkinsons Dis 2015;2015:916971.

30. Wang C, Cai Y, Gu Z, et al. Clinical profiles of Parkinson's disease associated with common leucine-rich repeat kinase 2 and glucocerebrosidase genetic variants in Chinese individuals. Neurobiol Aging 2014;35:725.e721-726.

31. Lesage S, Anheim M, Condroyer C, et al. Large-scale screening of the Gaucher's disease-related glucocerebrosidase gene in Europeans with Parkinson's disease. Hum Mol Genet 2011;20:202-210.

32. Gan-Or Z, Bar-Shira A, Mirelman A, et al. LRRK2 and GBA mutations differentially affect the initial presentation of Parkinson disease. Neurogenetics 2010;11:121-125.

33. Mullin S, Smith L, Lee K, et al. Ambroxol for the treatment of patients with Parkinson disease with and without glucocerebrosidase gene mutations: a nonrandomized, noncontrolled trial. JAMA Neurol 2020;77:427-434.

34. Bjornestad A, Tysnes OB, Larsen JP, Alves G. Loss of independence in early Parkinson disease: a 5-year population-based incident cohort study. Neurology 2016;87: 1599-1606. 


\section{Neurology}

\section{Association of GBA Genotype With Motor and Functional Decline in Patients With Newly Diagnosed Parkinson Disease \\ Jodi Maple-Grødem, Ingvild Dalen, Ole-Bjørn Tysnes, et al.}

Neurology 2021;96; 1036-e1044 Published Online before print December 21, 2020

DOI 10.1212/WNL.0000000000011411

This information is current as of December 21, 2020

\section{Updated Information \&} Services

References

Citations

Subspecialty Collections

Permissions \& Licensing

Reprints including high resolution figures, can be found at: http://n.neurology.org/content/96/7/e1036.full

This article cites 34 articles, 5 of which you can access for free at: http://n.neurology.org/content/96/7/e1036.full\#ref-list-1

This article has been cited by 1 HighWire-hosted articles: http://n.neurology.org/content/96/7/e1036.full\#\#otherarticles

This article, along with others on similar topics, appears in the following collection(s):

Association studies in genetics

http://n.neurology.org/cgi/collection/association_studies_in_genetics Cohort studies

http://n.neurology.org/cgi/collection/cohort_studies

Parkinson's disease/Parkinsonism

http://n.neurology.org/cgi/collection/parkinsons_disease_parkinsonism

Information about reproducing this article in parts (figures,tables) or in its entirety can be found online at:

http://www.neurology.org/about/about_the_journal\#permissions

Information about ordering reprints can be found online:

http://n.neurology.org/subscribers/advertise

Neurology ${ }^{\circledR}$ is the official journal of the American Academy of Neurology. Published continuously since 1951 , it is now a weekly with 48 issues per year. Copyright Copyright (C) 2020 The Author(s). Published by Wolters Kluwer Health, Inc. on behalf of the American Academy of Neurology.. All rights reserved. Print ISSN: 0028-3878. Online ISSN: 1526-632X.

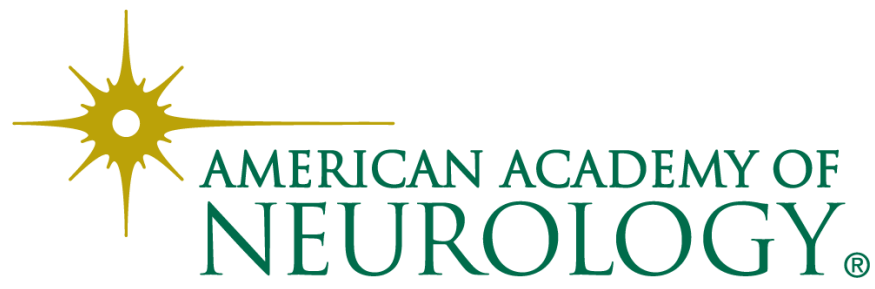

